\title{
HYDRAULIC AMPLIFICATION DEVICES FOR MICROSCALE ACTUATION
}

\author{
J.L. Steyn , H.Q. Li, D.C. Roberts, K.T. Turner, O. Yaglioglu, Y.-H. Su, \\ M.A. Schmidt, S.M. Spearing, N.W. Hagood \\ Massachusetts Institute of Technology \\ Cambridge, MA 02139 \\ R. Mlcak \\ Boston MicroSystems, Inc. \\ Woburn, MA 01801
}

\begin{abstract}
Hydraulic amplification devices for stroke amplification in MEMS devices have been designed, fabricated and evaluated experimentally. This work investigates the feasibility of using the concept of hydraulic amplification as a means of increasing the actuation stroke of a piezoelectric actuator in a microfabricated actuation system. The important aspects relating to the design of these devices are presented. This paper further provides a brief overview of the fabrication process used and then proceeds to address the topics related to the successful filling and sealing of small-scale hydraulic couplers. Both static and dynamic sealing techniques were used. Static tests were performed and stroke amplification ratios as high as 50:1 were obtained, with the peakto-peak amplified stroke being approximately $25 \mu \mathrm{m}$. Results from a piezoelectrically driven hydraulic amplifier are also presented and show resonant behavior at approximately $5 \mathrm{kHz}$, proving that high-frequency, high-force, large-stroke actuation can be performed on the microscale using piezoelectric hydraulic amplification.
\end{abstract}

\section{INTRODUCTION}

Relying on Pascal's law, hydraulic amplification can be seen as a conceptually elegant means of increasing either the stroke or the force of an actuator. No mechanical linkages or mechanisms are used, and there are only two moving parts - the two pistons. The actuator can take any form, but in this work we are primarily concerned with a piezoelectric actuator requiring stroke amplification.

The work presented here forms part of a larger program at the Massachusetts Institute of Technology to develop piezoelectric MicroHydraulic Transducer (MHT) technologies. The goal of MHT technology is to use hydraulics in conjunction with piezoelectric materials to produce hydraulic pumps or generators for actuation and power generation applications. It is beneficial to operate the piezoelectric material at high frequencies for maximum power output [1]. High frequency operation requires mechanical systems with high natural frequencies if quasi-static operation is desired. The use of fabrication technologies associated with MicroElectroMechanical Systems (MEMS) presents the opportunity to build small hydraulic machines capable of operating quasi-statically at high frequencies. In addition, the small scale results in increased power densities.

A 3-dimensional section view of the full MHT device being developed is shown in Figure 1. This device has a piezoelectrically driven piston and two piezoelectrically driven active valves. The use of so-called "active" valves instead of the typical "passive" check valves used in pumps allows for bi-directional operation, enabling this device to operate as both a pump and a generator. Both the main piston and the two valves employ a piezoelectric micro-actuator as described by Roberts et al [2].

The active valves (see [3]) require large stroke operation in the $\mathrm{kHz}$ range of frequencies. In addition, the device shown in Figure 1 is designed to operate at pressures as high as $2 \mathrm{MPa}$, and therefore the valves should also be able to operate at these pressures. Piezoelectric materials have sufficient actuation force and bandwidth to drive these valves, but are lacking in stroke. For this device, hydraulic amplification was chosen as the most feasible means of increasing the uniaxial actuation of a piezoelectric element.

Macroscale examples of hydraulic amplifiers exist. (See, e.g. $[4,5])$. This work proceeds to present, for the first time, hydraulic amplification as a general means of stroke amplification for use in MEMS, with the use of the basic principle extending beyond that of piezoelectric valve operation. The relevant issues pertaining to the development of such devices are discussed, and experimental results are presented.

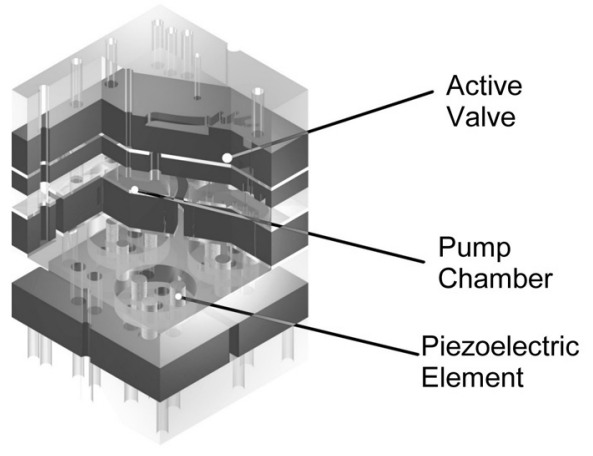

Figure 1. A $3 D$ section view of the full MicroHydraulic Transducer device with two hydraulically amplified piezoelectrically driven active valves and a piezoelectrically driven main piston.

\section{DEVICE LAYOUT AND DESIGN CONSIDERATIONS}

Figures 2 and 3 schematically show the layout of the devices that were evaluated. Figure 2 depicts a pressure operated hydraulic amplifier, and Figure 3 a piezoelectrically driven one.

Travel support has been generously provided by the Transducers Research Foundation and by the DARPA MEMS and DARPA BioFlips programs. 
Both devices consist of nine layers - five layers, L2, L4, L5, L7 and L8, are silicon layers, and the other four, L1, L3, L6 and L9, are borosilicate glass. In both devices, a short stroke motion of the large piston in layers 4 and 5 is amplified to a large stroke motion of the small piston in layer 7. All pistons are formed using Silicon On Insulator (SOI) substrates, where the SOI device layer is used as an annular membrane tether to attach the piston to the device structure and to act as a flexural seal.

In the process of the designing these devices, the following aspects were considered: (a) The stiffness and strength of the membrane tethers. Within the constraints of available SOI thicknesses, device area available, manufacturing capabilities and the required deflections, the membranes were designed for maximum strength and minimum added compliance to the hydraulic amplification chamber (HAC). (b) The added compliance of the remaining device structure. By use of finite element analysis, suitable dimensions for the silicon and glass support structures were defined to enable a compact yet stiff device. (c) The height of the HAC. This chamber, formed in L6, was designed for minimum volume without introducing unnecessary squeeze film damping with a chamber height that is too low.

The results of both a pressure-operated, as well as a piezoelectrically driven hydraulic amplifier are presented here. Both devices had a large piston of diameter $6.8 \mathrm{~mm}$ with a tether width of $225 \mu \mathrm{m}$, and a small piston diameter of $0.51 \mathrm{~mm}$ with corresponding tether width of $442 \mu \mathrm{m}$. This gives a theoretical stroke amplification ratio of between 40:1 and 50:1, when taking into account all the relevant compliances in the system. Due to geometric nonlinearities in the small piston structure, the amplification ratio is not constant for the full range of travel of the two pistons.

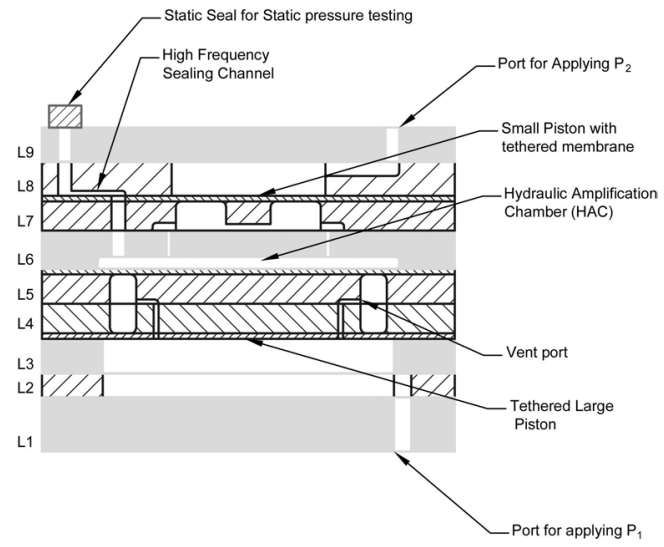

Figure 2. Static, pressure operated hydraulic amplifier.

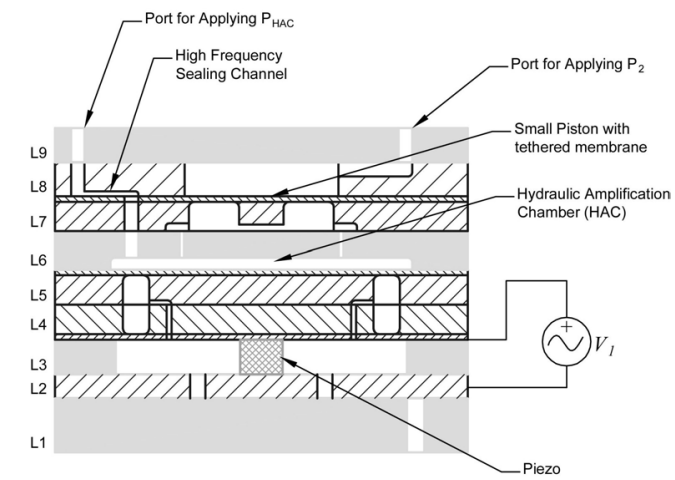

Figure 3. Dynamic, piezoelectrically driven hydraulic amplifier.

\section{FABRICATION OVERVIEW}

The silicon layers were bulk micromachined using Deep Reactive Ion Etching (DRIE). All glass layers (Pyrex ${ }^{\mathrm{TM}} 7740$ ) were ultrasonically machined, on the wafer scale. In the case of Layer 6, a $200 \mu \mathrm{m}$ deep recess was made to form the HAC.

Wafer-level Si-Si fusion bonding was used to bond L4 to L5, as well as L7 to L8. Wafer-level anodic bonding was used to bond L6 to the L4-5 stack and also to bond L1 to L2. All other bonds were anodic bonds performed on the die level. For the piezoelectric device shown in Figure 3, a small bulk piezoelectric element was integrated in the final anodic bonding step, as described in [2].

Figure 4 shows the device subassemblies, with Figure 5 showing a completed device. The final device is a nine-layer Siglass sandwich structure and has dimensions of 20x20x10mm.

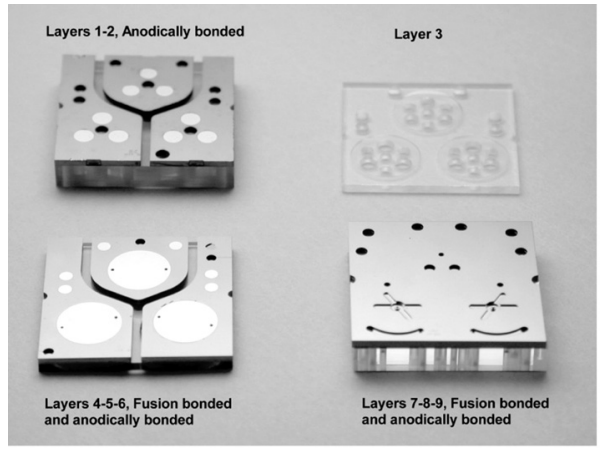

Figure 4: Subassemblies of the nine-layer hydraulic amplification device. Various unique fabrication techniques were used to produce these dies.

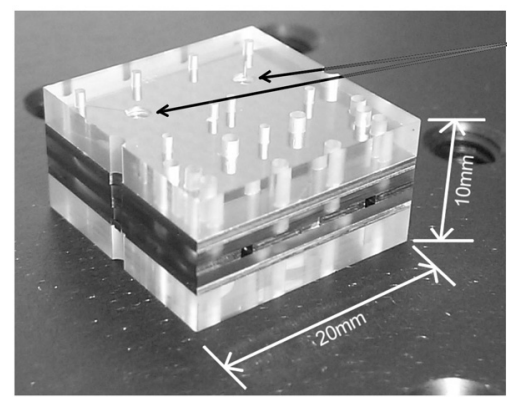

Two small pistons of the two hydraulic amplifiers
contained in this contained in
assembly.

Figure 5: Assembled hydraulic amplification device. Each such stack contains two independent hydraulic amplifiers.

\section{FILLING TECHNIQUES}

Effective hydraulic amplification relies on bubble-free filling of the HAC. To achieve this, a process was developed where the HAC is first evacuated and purged with the vapor of the hydraulic fluid. Afterwards, the chamber is filled with the fluid itself. This process ensured that any entrapped gases in the HAC would mostly consist of the vapor of the hydraulic fluid.

This method of vapor purging prior to filling required that the hydraulic fluid itself had a sufficiently high vapor pressure. The fluid of choice was hexamethyldisiloxane, with a viscosity at room temperature of $\sim 0.65 \mathrm{cst}$, and a vapor pressure of $6.7 \mathrm{kPa}$. Using this silicone oil, bubble free filling of the $\mathrm{HAC}$ was attained through a $10 \mu \mathrm{m} \times 10 \mu \mathrm{m}$ by $1000 \mu \mathrm{m}$ long filling channel. 


\section{SEALING TECHNIQUES}

Two methods were used for sealing the hydraulic amplifier. The first method, used for the pressure operated "static" devices, relied on a $125 \mu \mathrm{m}$ thick PTFE film backed by a brass plate and preloaded with an o-ring. This seal was placed over the filling hole in Layer 9. Minimal seal compression and creep was observed, and therefore the pressure inside the HAC remained unaffected.

For the piezoelectrically driven dynamic hydraulic amplifier, a "dynamic seal" was used. This seal consisted of a small (10 $\mu \mathrm{mx} 10 \mu \mathrm{m}$ cross section, $1000 \mu \mathrm{m}$ long) channel that acted as a fluid low pass filter. By using this sealing technique, it was possible to set the steady-state pressure inside of the HAC to a desired value using an outside pressure source, whilst maintaining high-frequency pressure fluctuations due to the piezoelectric actuation inside the HAC. Figure 6 illustrates the effectiveness of this channel. When driving the large piston, and observing the motion of the small piston, rolloff is detected at a frequency of $0.1 \mathrm{~Hz}$, well below the $\mathrm{kHz}$ operating frequencies of the device.

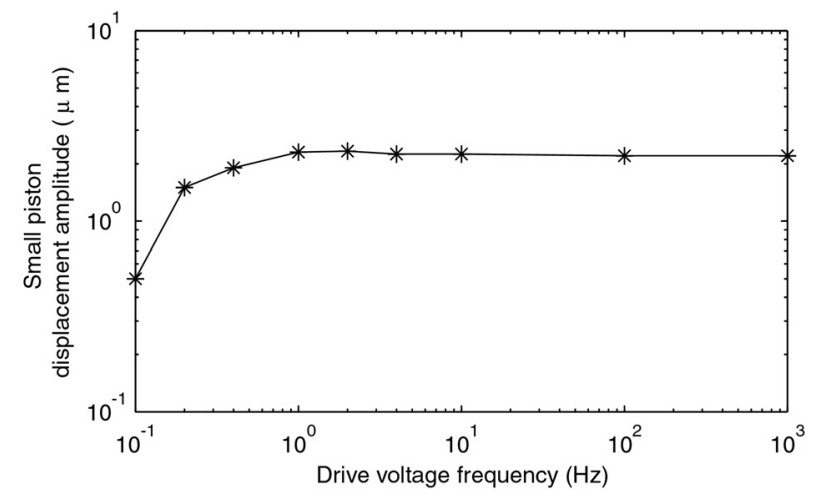

Figure 6: Frequency response of the dynamic seal. This seal acts as a low pass filter to the $H A C$.

\section{STATIC HYDRAULIC AMPLIFICATION}

Static, pressure-operated hydraulic amplification tests were performed to evaluate the ability of the hydraulic amplification chamber to couple the motion of the two pistons. Pressures were applied to the large piston $\left(P_{l}\right)$ and the small piston $\left(P_{2}\right) . P_{l}$ was varied from 0 to $200 \mathrm{kPa}$ for preset pressures of $P_{2}=0,53,105$, 153,206 and $253 \mathrm{kPa}$. Loading the HAC in this manner made it possible to prove the stiffness of the coupler. Using a laser vibrometry system, the displacements of the small and large pistons were measured. The results of these tests are shown in Figures 7 and 8 . From these results, one can calculate the amplification ratio (Figure 9), defined as the ratio of the difference in displacement of the small piston versus that of the large piston.

Figure 7 shows discrepancies between the predicted and actual motion. This is due to additional compliance in the HAC, which could be caused by a small bubble in the fluid in this particular case. The good agreement on the results in Figure 8, for the small piston, can be explained by the fact that the differential pressure across the large piston is not very large for the deflections seen. Therefore, the pressure on the large piston, $P_{l}$, is approximately equal to the pressure in the HAC, $P_{H A C}$. Therefore, Figure 8 is almost equivalent to a series of pressure-deflection tests on the small piston structure alone. Figure 9 shows similar

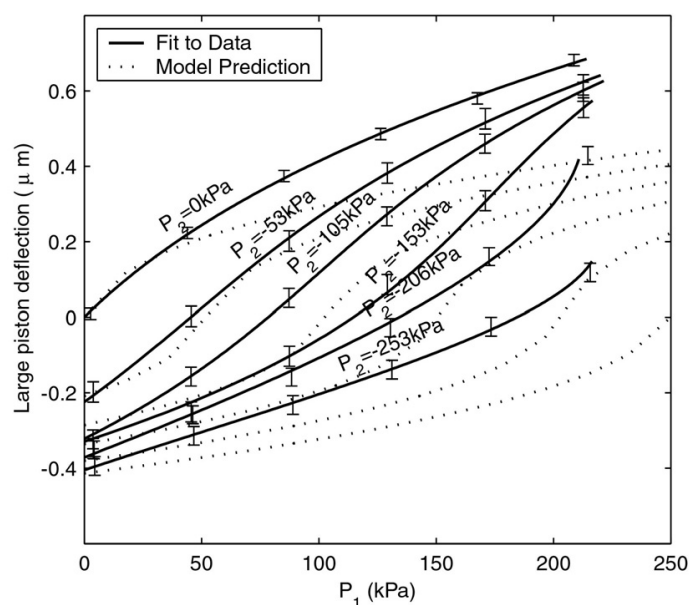

Figure 7: Motion of the large piston under applied pressure on both the large piston $\left(P_{1}\right)$, and the small piston $\left(P_{2}\right)$.

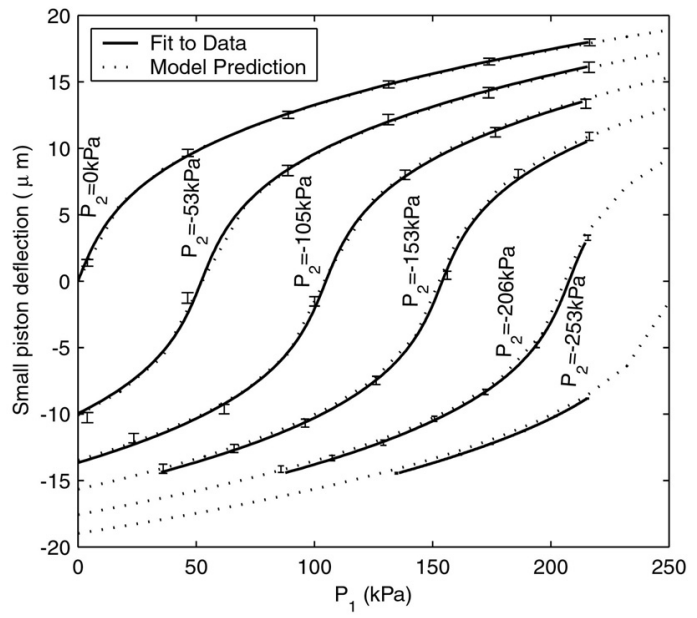

Figure 8: Motion of the small piston under applied pressure on both the large piston $\left(P_{1}\right)$, and the small piston $\left(P_{2}\right)$.

trends for both the predicted and measured amplification ratios, with the exception being the case where $P_{2}=0 \mathrm{kPa}$.

\section{VERIFICATION OF DYNAMIC OPERATION}

To prove that the hydraulic amplifier can function as intended - that is, to amplify the stroke of a piezoelectric actuation element tests were performed on a piezoelectrically driven hydraulic amplifier as was shown schematically in Figure 3. Figure 10 is an amplitude spectrum of the motion of the small piston, when excited using a sweep excitation on the piezoelectrically driven large piston. A first resonance of approximately $10 \mathrm{kHz}$ was observed when using gas to pressurize the small piston. When liquid was used on top of the small piston, this frequency reduced to approximately $5 \mathrm{kHz}$ due to the added mass effect of the fluid on the small piston.

Figure 11 shows the behavior of the small and large pistons for various excitation voltages at $1 \mathrm{kHz}$ sinusoidal excitation In this case, the voltage replaces the applied large piston pressure used to create Figures 7 and 8 . In the case of the dynamic hydraulic amplifier, much better correlation is seen between the predicted and measured results, most likely due to improved filling of the HAC. The same holds for the amplification ratio, depicted 


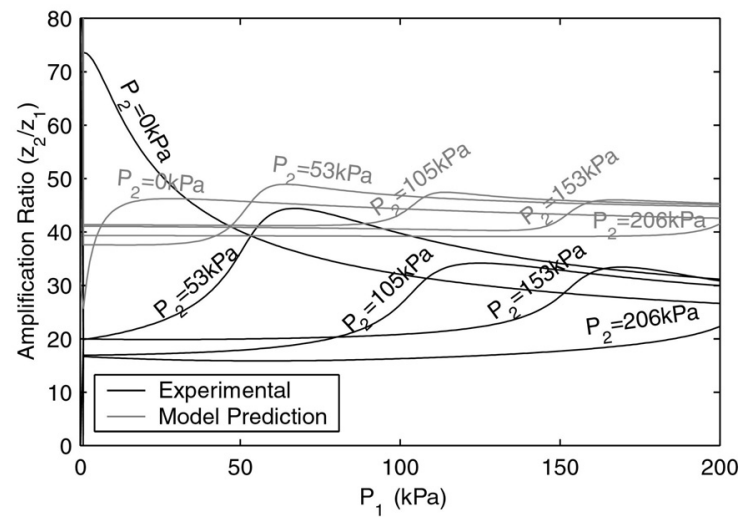

Figure 9: Amplification ratio for the static hydraulic amplifier.

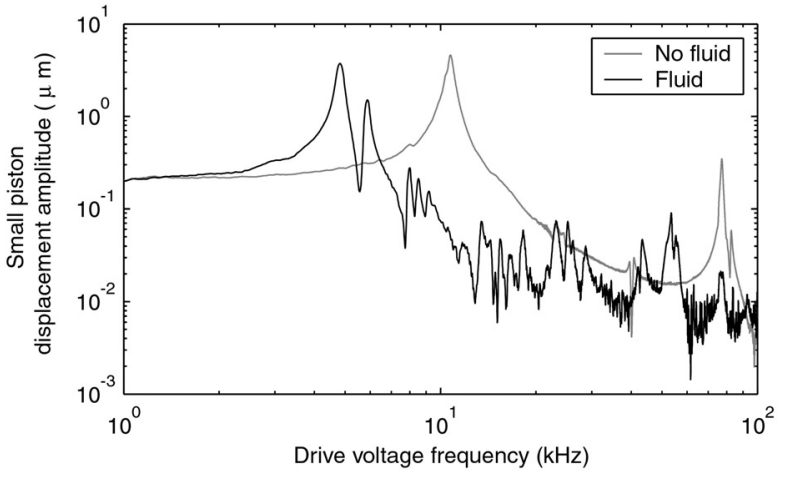

Figure 10: Displacement spectrum of the small piston. Excitation was 5 Vp-p.

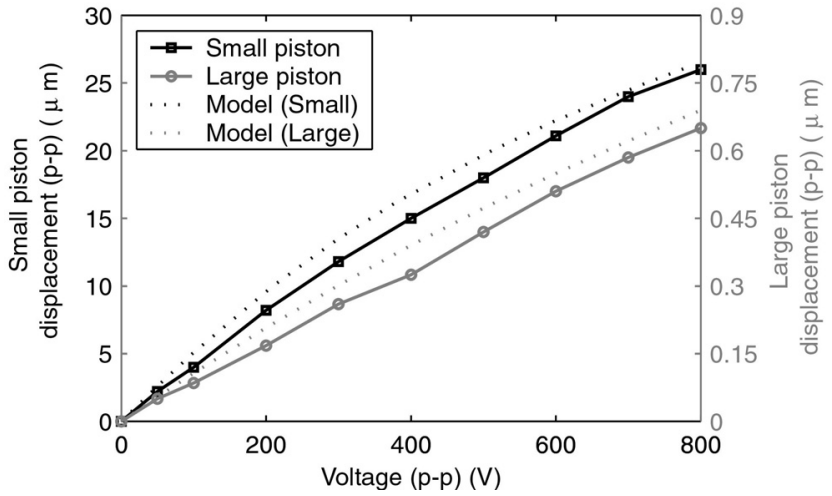

Figure 11: Displacement of the small and large pistons of the dynamic hydraulic amplifier for various excitation voltages. Sinusoidal excitation at $1 \mathrm{kHz}$.

in Figure 12. Note that there is acceptable agreement between the amplification ratios observed during static and dynamic actuation.

\section{CONCLUSIONS}

In this work we have developed and evaluated microfabricated hydraulic amplification devices. The important issues relating to the design of such devices on the microscale have been addressed. We have also demonstrated that filling and sealing of microfabricated dead volumes with hydraulic fluid is feasible, and stiff hydraulic couplers can be produced using the techniques described here. The experimental results show that microfabricated hydraulic amplification devices can have

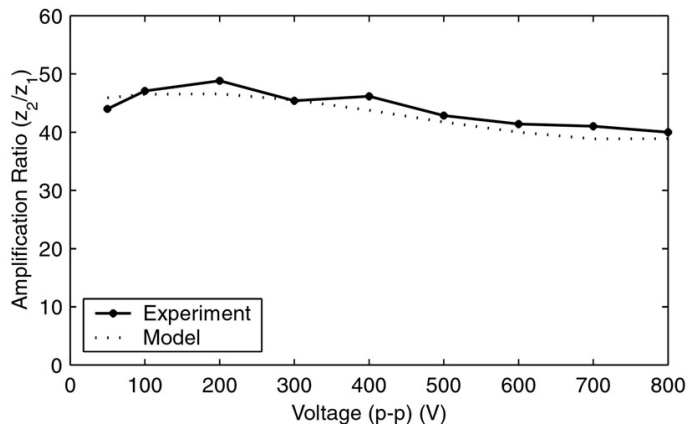

Figure 12: Amplification ratio results for the piezoelectric hydraulic amplifier.

sufficient stiffness and actuation authority for application in highpressure microvalves and other types of micropositioning devices. It has also been shown that these devices can have sufficient bandwidth to allow for high frequency operation, with large amplification ratios.

\section{ACKNOWLEDGMENTS}

All microfabrication was performed at the MIT Microsystems Technology Laboratories (MTL). The authors also wish to acknowledge the group of Prof. Liou at the University of Nebraska for all the Au-Sn sputter deposited films used to incorporate the piezoelectric elements. This work was performed under ONR grants N00014-01-1-0857 and N00014-97-1-0880 and DARPA grant DAAG55-98-1-0361.

\section{REFERENCES}

1. N.W. Hagood IV, et al. "Micro-hydraulic Transducer Technology for Actuation and Power Generation", SPIE $7^{\text {th }}$ International Symposium on Smart Structures and Materials, Newport Beach, CA, March 5-9, 2000.

2. D.C. Roberts et al, "A High Frequency, High Stiffness Piezoelectric Micro-Actuator for Hydraulic Applications," Proceedings of the 11th International Conference on Solid-State Sensors and Actuators, Munich, Germany, June 10-14, 2001, pp. 686-689.

3. D.C. Roberts et al, "Modeling, Design, and Simulation of a Piezoelectrically Driven Microvalve for High Pressure, High Frequency Applications," Proceedings of the SPIE 8th International Symposium on Smart Structures and Materials, Newport Beach, CA, March 5-8, 2001, Vol. 4327, pp. 366-380.

4. P. Tang, et al. "Combined Piezoelectric-Hydraulic Actuator Based Active Vibration Control for Rotordynamic System", Trans. of the ASME - Journal of Vibration and Acoustics, July 1995, Vol. 177, pp285-293

5. J. Garcia-Bonito, et al. "A novel high-displacement piezoelectric actuator for active vibration control", Smart Materials and Structures, Vol 7 1998, pp31-42, IOP

6. H.Q. Li, et al, "A High Frequency, High Flow Rate Piezoelectrically Driven MEMS Micropump", Technical Digest of the 2000 Solid-State Sensor and Actuator Workshop, Hilton Head, SC, June 2000

7. J.L. Steyn, "Hydraulic Amplification for Actuation in MicroElectroMechanical Systems", Master's Thesis, MIT, 2002

8. D.C. Roberts, "Design, modeling, fabrication and testing of a piezoelectric microvalve for high pressure, high frequency hydraulic applications", PhD Thesis, MIT, 2002 